\title{
Triterpenoids from Phyllanthus acidus (L.) Skeels
}

\author{
Duong Thuc Huy ${ }^{1}$, Nguyen Huu Hung ${ }^{2}$, Nguyen Thi Anh Tuyet ${ }^{1}$, Bui Xuan Hao ${ }^{1}$
}

\begin{abstract}
The genus Phyllanthus (Phyllanthaceae) includes more than 900 plant species found in tropical and subtropical regions. Many of these species are widely used in folk medicine. The leaves, roots, and stem bark of Phyllanthus acidus (L.) Skeels have been used in Vietnamese folk medicine as an antibacterial, antiviral, analgesic, anti-inflammatory, neuroprotective, hepatoprotective, antifibrotic. From the ethanol extract of the roots of Phyllanthus acidus (L.) Skeels growing in Binh Thuan province, six compounds phyllanthol (1), glochidone (2), lupeol (3), glochidonol (4), $\alpha$-lupene (5), and spruceanol (6) were isolated. Their structures were established by extensive spectroscopic analysis as well as comparison with NMR data in the literatures. This is the first time that compounds 4-6 were found in Phyllanthus acidus (L.) Skeels.
\end{abstract}

Keywords_Phyllanthus acidus (L.) Skeels, lupane, phyllanthol, triterpene

\section{INTRODUCTION}

$\mathrm{P}^{\mathrm{r}}$ revious studies on chemical constituents of Phyllanthus acidus (L.) Skeels resulted in the discovery of various natural products such as triterpenes, phytosterols, phenolic compounds, and norbisabolane-type sesquiterpenes [1-3]. Among them, norbisabolane serquiterpenoids displayed strong anti-viral (hepatitis B) effect [3]. Our previous study on the stem bark of Phyllanthus acidus (L.) Skeels led to the isolation of three compounds [4].

This paper reports details of the isolation of six compounds from the roots of Phyllanthus acidus (L.) Skeels, including phyllanthol (1), glochidone

Received: 15-8-2017; Accepted: 12-9-2017; Published: 30-8-2018

Duong Thuc Huy, Nguyen Thi Anh Tuyet, Bui Xuan Hao* - Ho Chi Minh City University of Pedagogy.

Nguyen Huu Hung - Nguyen Tat Thanh University

*Email: buixuanhaodhsp@gmail.com
(2), lupeol (3), glochidonol (4), $\alpha$-lupene A (5), and spruceanol (6). Their structure were elucidated on the basis of NMR analysis.

\section{MATERIALS AND METHODS}

\section{General experimental procedures}

The NMR spectra were measured on a Bruker Avance III $\left(500 \mathrm{MHz}\right.$ for ${ }^{1} \mathrm{H}$ NMR and $125 \mathrm{MHz}$ for ${ }^{13} \mathrm{C}$ NMR) spectrometer with TMS as internal standard. Proton chemical shifts were referenced to the solvent residual signal of $\mathrm{CDCl}_{3}$ at $\delta_{\mathrm{H}}$ 7.26. The ${ }^{13} \mathrm{C}-\mathrm{NMR}$ spectra were referenced to the peak of $\mathrm{CDCl}_{3}$ at $\delta_{\mathrm{C}}$ 77.2. Gravity column chromatography was performed with Silica gel $60(0.040-0.063 \mathrm{~mm}$, Himedia).

\section{Plant material}

Phyllanthus acidus (L.) Skeels was collected in Ham Thuan Nam district, Binh Thuan province. This plant was identified by Msc. Hoang Viet, Faculty of Biology, University of Science, VNU HCM. A voucher specimen (No UP-B01) was deposited in the herbarium of the Department of Organic Chemistry, Faculty of Chemistry, Ho Chi Minh University of Pedagogy.

\section{Extraction and isolation}

The ground root material $(20.0 \mathrm{~kg})$ was extracted with $95 \%$ ethanol under reflux ( $3 \times 10$ L) and the filtrated solution was concentrated under the reduced pressure to obtain the crude extract $(1 \mathrm{~kg})$. A half of this crude extract $(500.0 \mathrm{~g})$ was applied to normal phase silica gel column chromatography eluted with increasing polarity of ethyl acetate $/ n$-hexane ether ( 0 $100 \%)$ to afford the fractions $\mathbf{H 1}(2.0 \mathrm{~g}), \mathbf{H} 2$ (4.0g), H3 (2.1g), H4 (3.4g), and EA1 (67.0g). The remaining residue was eluted with ethyl 
acetate: methanol (50:50) and (0:100) to afford the extracts EA2 $(85.0 \mathrm{~g})$ and Me $(285.0 \mathrm{~g})$.

Fraction H1 (2.0 g) was applied to silica gel column chromatography, eluted with $n$-hexane: ethyl acetate (9:1) to obtain five subfractions H1.1 (125.0mg), H1.2 (250.0 mg), H1.3 (152.0 mg), H1.4 (150.0mg), and H1.5 (1.1g).

Subfraction H1.2 was chromatographed, eluted with $n$-hexane: methanol (100:0.2) to obtain three subfractions H1.2.1 (60.0mg), H1.2.2 (55.0mg), and H1.2.3 (75.0mg). Subfraction H1.2.1 was rechromatographed, eluted with $n$ hexane: methanol $(100: 0.2)$ to afford three compounds $\mathbf{1}$ (6mg), 2 (30mg), and $\mathbf{5}(5 \mathrm{mg})$. Purifying the subfraction H1.2.3 by column chromatography, eluted with $n$-hexane: methanol $(100: 0.2)$ resulted in two compounds, $3(22.0 \mathrm{mg})$ and 4 (8.0mg). Subfraction H1.5 was washed many times by ethyl acetate to afford compound 1 (800mg). Fraction EA2 was suspended in $\mathrm{H}_{2} \mathrm{O}$ $(0.5 \mathrm{~L})$ and partitioned with EtOAc $(3 \times 0.5 \mathrm{~L})$ to obtain the EtOAc-soluble subfraction E0 (7.0g) and remaining aqueous fraction $(70.0 \mathrm{~g})$. The subfraction E0 was concentrated then applied to silica gel column chromatography, eluted with chloroform: methanol: water $(4: 0.9: 0.1)$ to obtain five subfractions E0.1 - E0.5. Subfraction E0.1 $(1.16 \mathrm{~g})$ was chromatographed, eluted with petroleum ether: ethyl acetate: acetic acid (5:1:0.2) to obtain nineteen subfractions E0.1.1 E0.1.19. Purifying the subfraction E0.1.14 (46.0mg) by column chromatography, eluted with petroleum ether: chloroform: methanol (1:8:0.2) resulted in compound 6 (8.8mg).
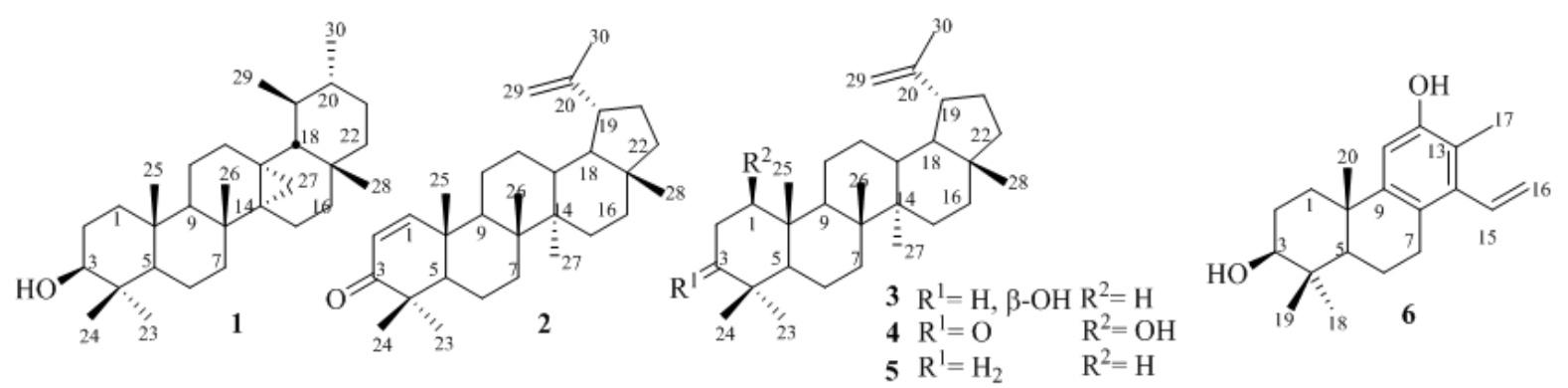

Fig. 1. Chemical structures of 1-6

Table 1. ${ }^{13} \mathrm{C}-\mathrm{NMR}$ data of $1-6\left(\mathrm{CDCl}_{3}\right)$

\begin{tabular}{|c|c|c|c|c|c|c|c|c|c|c|c|c|c|}
\hline No & 1 & 2 & 3 & 4 & 5 & 6 & No & 1 & 2 & 3 & 4 & 5 & 6 \\
\hline 1 & 38.5 & 160.1 & 38.2 & 79.6 & 40.1 & 37.4 & 16 & 27.9 & 35.0 & 35.8 & 35.5 & 35.7 & 119.7 \\
\hline 2 & 29.4 & 124.5 & 25.3 & 45.1 & 19.4 & 28.3 & 17 & 31.1 & 42.6 & 43.2 & 43.0 & 43.1 & 13.0 \\
\hline 3 & 79.1 & 203.9 & 79.3 & 215.6 & 42.2 & 78.9 & 18 & 54.0 & 47.7 & 48.5 & 48.3 & 48.4 & 28.2 \\
\hline 4 & 38.8 & 42.9 & 38.9 & 47.1 & 33.3 & 38.8 & 19 & 40.8 & 47.3 & 48.1 & 47.9 & 48.1 & 15.3 \\
\hline 5 & 55.7 & 52.8 & 55.5 & 51.4 & 55.1 & 49.3 & 20 & 37.3 & 150.1 & 151.1 & 150.7 & 151.0 & 24.8 \\
\hline 6 & 18.1 & 18.9 & 18.5 & 19.6 & 19.4 & 19.2 & 21 & 29.7 & 29.2 & 30.0 & 29.8 & 30.0 & \\
\hline 7 & 38.4 & 33.2 & 34.5 & 35.5 & 34.3 & 29.8 & 22 & 42.0 & 39.8 & 40.2 & 40.0 & 39.8 & \\
\hline 8 & 37.0 & 41.2 & 41.0 & 40.0 & 40.9 & 125.3 & 23 & 27.3 & 27.4 & 28.2 & 27.9 & 33.7 & \\
\hline 9 & 50.1 & 43.9 & 50.6 & 50.7 & 49.9 & 147.9 & 24 & 15.3 & 21.2 & 15.6 & 19.9 & 21.6 & \\
\hline 10 & 37.3 & 39.0 & 37.3 & 43.0 & 37.8 & 38.8 & 25 & 16.0 & 18.5 & 16.3 & 11.8 & 15.9 & \\
\hline 11 & 17.6 & 20.5 & 21.1 & 23.1 & 21.2 & 109.5 & 26 & 17.9 & 16.1 & 16.2 & 16.0 & 16.1 & \\
\hline 12 & 35.2 & 24.6 & 27.5 & 25.2 & 25.3 & 151.9 & 27 & 13.3 & 14.1 & 14.7 & 14.5 & 14.6 & \\
\hline 13 & 26.6 & 37.5 & 39.0 & 38.0 & 38.3 & 119.0 & 28 & 28.2 & 17.8 & 18.2 & 18.0 & 18.2 & \\
\hline 14 & 32.2 & 42.6 & 43.0 & 43.0 & 43.0 & 139.2 & 29 & 18.0 & 109.7 & 109.5 & 109.4 & 109.5 & \\
\hline 15 & 21.3 & 26.9 & 27.6 & 27.5 & 27.6 & 135.5 & 30 & 20.7 & 18.9 & 19.5 & 19.3 & 19.8 & \\
\hline
\end{tabular}

Phyllanthol (1): White amorphous powder. The ${ }^{1} \mathrm{H}-\mathrm{NMR}$ data $\left(\delta\right.$ in ppm, $\left.\mathrm{CDCl}_{3}\right): 3.19(1 \mathrm{H}$, $d d, 11.0,5.0 \mathrm{~Hz}, \mathrm{H}-3), 0.96(3 \mathrm{H}, s, \mathrm{H}-23), 0.77$ $(3 \mathrm{H}, s, \mathrm{H}-24), 0.86(3 \mathrm{H}, s, \mathrm{H}-25), 1.14(3 \mathrm{H}, s, \mathrm{H}-$ 26), $0.01(1 \mathrm{H}, d, 5.5 \mathrm{~Hz}, \mathrm{H}-27 \mathrm{a}), 0.66(1 \mathrm{H}, d, 5.5$
Hz, H-27b), 0.90 (3H, s, H-28), 0.94 (3H, $d, 6.0$ $\mathrm{Hz}, \mathrm{H}-29), 0.87(3 \mathrm{H}, d, 6.0 \mathrm{~Hz}, \mathrm{H}-30)$. The ${ }^{13} \mathrm{C}-$ NMR data $\left(\mathrm{CDCl}_{3}\right)$ : see Table 1. These spectroscopic data were suitable with those reported in the literature [5]. 
Glochidone (2): Colorless oil. The ${ }^{1} \mathrm{H}-\mathrm{NMR}$ data $\left(\delta\right.$ in ppm, $\left.\mathrm{CDCl}_{3}\right): 7.10(1 \mathrm{H}, d, 10.0 \mathrm{~Hz}, \mathrm{H}-$ 1), $5.79(1 \mathrm{H}, d, 10.0 \mathrm{~Hz}, \mathrm{H}-2), 2.40(1 \mathrm{H}, t d, 11.0$, $6.0 \mathrm{~Hz}, \mathrm{H}-19), 1.06$ (3H, $s, \mathrm{H}-23), 0.95$ (3H, $s, \mathrm{H}-$ 24), $1.08(3 \mathrm{H}, s, \mathrm{H}-25), 1.12(3 \mathrm{H}, s, \mathrm{H}-26), 1.11$ $(3 \mathrm{H}, s, \mathrm{H}-27), 0.80(3 \mathrm{H}, s, \mathrm{H}-28), 4.70(1 \mathrm{H}, d, 2.0$ $\mathrm{Hz}, \mathrm{H}-29 \mathrm{a}), 4.59(1 \mathrm{H}, d, 2.0 \mathrm{~Hz}, \mathrm{H}-29 \mathrm{~b}), 1.69$ $(3 \mathrm{H}, s, \mathrm{H}-30)$. The ${ }^{13} \mathrm{C}-\mathrm{NMR}$ data $\left(\mathrm{CDCl}_{3}\right)$ : see Table 1. These spectroscopic data were suitable with those reported in the literature [6].

Lupeol (3): White amorphous powder. The ${ }^{1} \mathrm{H}-\mathrm{NMR}$ data $\left(\delta\right.$ in ppm, $\left.\mathrm{CDCl}_{3}\right): 3.16(1 \mathrm{H}, d d$, $11.0,4.8 \mathrm{~Hz}, \mathrm{H}-3), 2.36(1 \mathrm{H}, t d, 11.0,5.5 \mathrm{~Hz}, \mathrm{H}-$ 19), 0.95 (3H, $s, \mathrm{H}-23), 0.75$ (3H, $s, \mathrm{H}-24), 0.82$ $(3 \mathrm{H}, s, \mathrm{H}-25) 1.02(3 \mathrm{H}, s, \mathrm{H}-26), 0.93(3 \mathrm{H}, s, \mathrm{H}-$ 27), $0.78(3 \mathrm{H}, s, \mathrm{H}-28), 4.68(1 \mathrm{H}, d, 2.0 \mathrm{~Hz}, \mathrm{H}-$ 29a), $4.56(1 \mathrm{H}, d d, 2.5,1.5 \mathrm{~Hz}, \mathrm{H}-29 \mathrm{~b}), 1.67(3 \mathrm{H}$, $s$, H-30). The ${ }^{13} \mathrm{C}-\mathrm{NMR}$ data $\left(\mathrm{CDCl}_{3}\right)$ : see Table 1. These spectroscopic data were suitable with those reported in the literature [8].

Glochidonol (4): White amorphous powder. The ${ }^{1} \mathrm{H}-\mathrm{NMR}$ data $\left(\delta\right.$ in ppm, $\left.\mathrm{CDCl}_{3}\right): 3.90(1 \mathrm{H}$, $d d, 8.0,3.5 \mathrm{~Hz}, \mathrm{H}-1), 3.00(1 \mathrm{H}, d d, 14.5,8.5 \mathrm{~Hz}$, $\mathrm{H}-2 \mathrm{a}), 2.23(1 \mathrm{H}, d d, 14.5,3.5 \mathrm{~Hz}, \mathrm{H}-2 \mathrm{e}), 2.37$ $(1 \mathrm{H}, t d, 11.5,5.5 \mathrm{~Hz}, \mathrm{H}-19), 1.03(3 \mathrm{H}, s, \mathrm{H}-23)$, 0.97 (3H, s, H-24), 0.83 (3H, s, H-25), $1.06(3 \mathrm{H}$, $s, \mathrm{H}-26), 1.06(3 \mathrm{H}, s, \mathrm{H}-27), 0.80(3 \mathrm{H}, s, \mathrm{H}-28)$, $4.68(1 \mathrm{H}, d, 2.0 \mathrm{~Hz}, \mathrm{H}-29 \mathrm{a}), 4.56(1 \mathrm{H}, d, 2.0 \mathrm{~Hz}$, $\mathrm{H}-29 \mathrm{~b}), 1.68(3 \mathrm{H}, s, \mathrm{H}-30)$. The ${ }^{13} \mathrm{C}-\mathrm{NMR}$ data $\left(\mathrm{CDCl}_{3}\right)$ : see Table 1 . These spectroscopic data were suitable with those reported in the literature [6].

$\boldsymbol{\alpha}$-Lupene (5): White amorphous powder. The ${ }^{1} \mathrm{H}-\mathrm{NMR}$ data ( $\delta$ in ppm, $\left.\mathrm{CDCl}_{3}\right): 1.03(3 \mathrm{H}, s$, $\mathrm{H}-23), 0.80(3 \mathrm{H}, s, \mathrm{H}-24), 0.96(3 \mathrm{H}, s, \mathrm{H}-25)$, 1.07 (3H, s, H-26), 0.93 (3H, s, H-27), $0.87(3 \mathrm{H}$, $s, \mathrm{H}-28), 4.69(1 \mathrm{H}, d, 2.5 \mathrm{~Hz}, \mathrm{H}-29 \mathrm{a}), 4.57(1 \mathrm{H}$, $d, 2.5 \mathrm{~Hz}, \mathrm{H}-29 \mathrm{~b}), 1.68(3 \mathrm{H}, s, \mathrm{H}-30)$. The ${ }^{13} \mathrm{C}-$ NMR data $\left(\mathrm{CDCl}_{3}\right)$ : see Table 1. These spectroscopic data were suitable with those reported in the literature $[7,8]$.

Spruceanol (6): White amorphous powder. The ${ }^{1} \mathrm{H}-\mathrm{NMR}$ data $\left(\delta\right.$ in $\left.\mathrm{ppm}, \mathrm{CDCl}_{3}\right): 2.23(1 \mathrm{H}$, $m, \mathrm{H}-1 \mathrm{e}), 1.75$ (1H, $m, \mathrm{H}-1 \mathrm{a}), 1.80$ (2H, $m, \mathrm{H}-2)$, $3.29(1 \mathrm{H}, d d, 11.5,4.5 \mathrm{~Hz}, \mathrm{H}-3), 1.29(1 \mathrm{H}, d d$, 2.0, $2.0 \mathrm{~Hz}, \mathrm{H}-5), 1.89$ (1H ddd, 13.5, 7.5, $1.0 \mathrm{~Hz}$,
$\mathrm{H}-6 \mathrm{e}), 1.67(1 \mathrm{H} d d d, 13.5,11.5,6.0 \mathrm{~Hz}, \mathrm{H}-6 \mathrm{a})$, $2.78(1 \mathrm{H} d d d, 17.5,6.0,1.0 \mathrm{~Hz}, \mathrm{H}-7 \mathrm{e}), 2.57(1 \mathrm{H}$, $d d d, 17.5,11.5,7.5 \mathrm{~Hz}, \mathrm{H}-7 \mathrm{a}), 6.67(1 \mathrm{H}, s, \mathrm{H}-11)$, $6.57(1 \mathrm{H}, d d, 17.5,11.0 \mathrm{~Hz}, \mathrm{H}-15), 5.53(1 \mathrm{H}, d d$, 11.0, $2.5 \mathrm{~Hz}, \mathrm{H}-16 \mathrm{a}), 5.16(1 \mathrm{H}, d d, 17.5,2.0 \mathrm{~Hz}$, $\mathrm{H}-16 \mathrm{~b}), 2.18$ (3H, s, H-17), $1.06(3 \mathrm{H}, s, \mathrm{H}-18)$, $0.88(3 \mathrm{H}, s, \mathrm{H}-19), 1.20(3 \mathrm{H}, s, \mathrm{H}-20)$. The ${ }^{13} \mathrm{C}-$ NMR data $\left(\mathrm{CDCl}_{3}\right)$ : see Table 1. These spectroscopic data were suitable with those reported in the literature [9].

\section{RESULTS AND DISCUSSION}

Phyllanthol (1) was isolated from P. acidus in the first time by Sengupta and Mukhopadhyay (1966) [10] and its NMR data was revised later by Ndlebe (2008) [5]. It was found in some Phyllanthus species such as $P$. engleri, $P$. sellowianus [1], and Phyllanthus polyanthus [5].

Lupane-type triterpenes as glochidone (2), lupeol (3), glochidonol (4), and $\alpha$-lupene (5) were found in many Phyllanthus plants [1]. Such compounds, for examples lupeol and glochidone showed good inhibition to enzyme acetylcholine esterase [11]. Nevertheless, glochidonol (4) and $\alpha$-lupene (5) have not been isolated from $P$. acidus. Glochidonol (4) exerted good inhibitory effect on Epstein-Barr virus early antigen (EBV-EA) induced by TPA [12].

Compound $\mathbf{6}$ was isolated as a white amorphous powder. The ${ }^{13} \mathrm{C}$-NMR spectrum (Table 1) displayed signals corresponding to twenty carbons, including five quaternary carbons, two quaternary carbons, one oxygenated methine, one aromatic methine, two olefinic methines, four methylenes, one methine, and four methyls. The ${ }^{1} \mathrm{H}-\mathrm{NMR}$ spectrum displayed signals corresponding to one aromatic proton $\mathrm{H}-11\left[\delta_{\mathrm{H}}\right.$ $6.67(1 \mathrm{H}, s)]$ and three olefinic protons $\mathrm{H}-15\left[\delta_{\mathrm{H}}\right.$ $6.57(1 \mathrm{H}, d d, 17.5,11.0 \mathrm{~Hz})], \mathrm{H}-16 \mathrm{a}\left[\delta_{\mathrm{H}} 5.53(1 \mathrm{H}\right.$, $d d, 11.0,2.5 \mathrm{~Hz})]$, and $\mathrm{H}-16 \mathrm{~b}\left[\delta_{\mathrm{H}} 5.16(1 \mathrm{H}, d d\right.$, $17.5,2.5 \mathrm{~Hz})$ ], which were representative for one vinyl group $\left(\mathrm{CH}_{2}=\mathrm{CH}-\right)$. Moreover, the ${ }^{1} \mathrm{H}-\mathrm{NMR}$ spectrum revealed four singlet methyl H-17 $\left(\delta_{\mathrm{H}}\right.$ $2.18), \mathrm{H}-18\left(\delta_{\mathrm{H}} 1.06\right), \mathrm{H}-19\left(\delta_{\mathrm{H}} 0.83\right)$, and $\mathrm{H}-20$ (1.20), one oxygenated methine $\mathrm{H}-3$ at $\delta_{\mathrm{H}} 3.29$ $(d d, 11.5,4.5 \mathrm{~Hz})$. The axial position of $\mathrm{H}-3$ ( $\delta=$ $3.29, J=11.5,4.5 \mathrm{~Hz})$ in the A-ring was 
determined on the basis of coupling constants. The HMBC spectrum confirmed the correlations between $\mathrm{H}-3$ and the C-4, C-18, H-18 and $\mathrm{H}-19$ to $\mathrm{C}-3$ and $\mathrm{C}-4$, indicating their vicinal positions in A-ring. Additionally, $\mathrm{HMBC}$ spectrum showed cross peaks of $\mathrm{H}-17$ and $\mathrm{H}-16$ to $\mathrm{C}-14$, of $\mathrm{H}-17$ and $\mathrm{H}-11$ to $\mathrm{C}-12$ indicating positions of $\mathrm{H}-11$, 12-OH and $\mathrm{H}-17$ in the $\mathrm{C}$ ring. Further analysis of HMBC spectrum confirmed the structure of $\mathbf{6}$, according to comparison of the NMR data of $\mathbf{6}$ to those of spruceanol in the literature [9]. So, the structrure of compound $\mathbf{6}$ was concluded as spruceanol. This is the first time the diterpenoid skeleton was reported in P. acidus.

\section{CONCLUSION}

Six known compounds were isolated from the ethanol extract of the roots of Phyllanthus acidus growing in Binh Thuan province. Phyllanthol (1) was isolated as a major compound of the $n$-hexane extract. Glochidonol (4), $\alpha$-lupene (5), and spruceanol (6) are reported in the plant Phyllanthus acidus. Further studies on this plant are in progress.

\section{REFERENCES}

[1]J.B. Calixto, A.R.S. Santos, V.C. Filbo, R.A. Yunes, "A review of the plants of the genus Phyllanthus: their chemistry, pharmacology, and therapeutic potential", Medicinal Research Reviews, vol. 18, no. 4, pp. 225-258, 1998.

[2]Y. Leeya, M.J. Mulvany, E.F. Queiroz, A. Marston, K. Hostettmann, C. Jansakul, "Hypotensive activity of an nbutanol extract and their purified compounds from leaves of Phyllanthus acidus (L.) Skeel in rats", European Journal of Pharmacology, vol. 649, pp. 301-313, 2010.

[3]J.J. Lv, S. Yu, Y.F. Wang, D. Wang, H.T. Zhu, R.R. Cheng, C.R. Yang, M. Xu, Y.J. Zhang, “Anti-hepatitus B virus norbisabolane sesquiterpenoids from Phyllanthus acidus and the establishment of their absolute configurations using theoretical calculations", Journal of
Organic Chemistry, vol. 79, no. 12, pp. 5432-5447, 2014.

[4]T.T. Nguyen, T.H. Duong, T.A.T. Nguyen, X.H. Bui, "Study on the chemical constituents of Phyllanthus acidus (Euphorbiaceae)", Journal of Science and Technology, vol. 52, no. 5A, pp. 156-161, 2014.

[5] V.J. Ndlebe, N.R. Crouch, D.A. Mulholland, "Triterpenoid from the African tree Phyllanthus polyanthus", Phytochemistry Letters, vol. 1, no. 11-17, 2008.

[6]W.A. Ayer, R.J. Flanagan, T. Reffstrup, "Metabolites of bird's nest fungi, new triterpenenoid carboxylic acids from Cyathus striatus and Cyathus pygmaeus", Tetrahedron, vol. 40, no. 11, pp. 2069-2082, 1984.

[7]E. Wenkert, G.V. Baddeley, I.R. Burfitt, L.N. Moreno, "Carbon-13 Nuclear magnetic resonance spectroscopy of naturally occurring substances LVII, triterpenes related to lupane and hopane", Organic Magnetic Resonance, vol. 11, no. 7, pp. 337-343, 1978.

[8]S.B. Mahato, A.P. Kundu, "13C NMR spectra of pentacyclic triterpenoids, a compilation and some salient features", Phytochemistry, vol. 37, no. 6, pp. 1517-1575, 1994.

[9] A.B. Alimboyoguen, D. Castro, K.A. Cruz, C. Shen, W. Li, C.Y. Ragasa, "Chemical constituents of the bark of Aleurites moluccana L. Willd, Journal of Chemical and Pharmaceutical Research, vol. 6, no. 5, pp. 1318-1320b, 2014.

[10] P. Sengupta, J. Mukhopadhyay, "Terpenoids and related compds. VII. Triterpenoids of Phyllanthus acidus", Phytochemistry, vol. 5, no. 3, pp. 531-534, 1966.

[11] B. Culhaogu, S.D. Hatipoglu, A.A. Donmez, G. Topcu, "Antioxidant and anticholinesterase activities of lupane triterpenoids and other constituents of Salvia trichoclada", Medicinal Research Reviews, vol. 24, pp. 3831-3837, 2015.

[12] R. Tanaka, Y. Kinouchi, S. Wada, H. Tokuda, "Potential anti-tumor promoting activity of lupane-type tritepenenoids from the stem bark of Glochidion zeylancium and Phyllanthus flexuosus", Planta Medica Letters, vol. 70, pp. 1234-1236, 2004. 


\title{
Thành phần hóa học rễ cây chùm ruột mọc ở tỉnh Bình Thuận
}

\author{
Dương Thúc Huy ${ }^{1}$, Nguyễn Hữu Hùng ${ }^{2}$, Nguyễn Thị Ánh Tuyết ${ }^{1}$, Bùi Xuân Hào ${ }^{1, *}$ \\ ${ }^{1}$ Trường Đại học Sư phạm TP. HCM; ${ }^{2}$ Trường Đại học Nguyễn Tất Thành \\ *Tác giả liên hệ: buixuanhaodhsp@gmail.com
}

Ngày nhận bản thảo: 15-08-2017; Ngày chấp nhận đăng: 12-09-2017; Ngày đăng: 30-8-2018

Tóm tắt-Chi Phyllanthus (Phyllanthaceae) bao gồm hơn 900 loài thực vật, được tìm thấy ở vùng nhiệt đới và cận nhiệt đới. Nhiều loài trong chi này được sử dụng rộng rãi trong y học dân gian. Trong y học cổ truyền Việt $\mathrm{Nam}$, lá, rễ và vỏ thân của loài Phyllanthus acidus (L.) Skeels đã được sử dụng để kháng khuẩn, kháng vi-rút, giảm đau, chống viêm, bảo vệ thần kinh, chống viêm gan. Từ dịch chiết ethanol của rễ cây chùm ruột mọc ở tỉnh Bình
Thuận, đã phân lập được sáu hợp chất là phyllanthol (1), glochidone (2), lupeol (3), glochidonol (4), $\alpha$-lupene (5), spruceanol (6). Cấu trúc của các hợp chất này được làm sáng tỏ bằng các phương pháp phổ cộng hưởng từ hạt nhân, cũng như so sánh với các tài liệu tham khảo. Đây là lần đầu tiên các hợp chất $4,5,6$ được phát hiện trong cây chùm ruột.

Tù̀ khóa-Phyllanthus acidus (L.) Skeels, lupane, phyllanthol, diterpene 